\title{
ARTICLE Secondary necrotic neutrophils release interleukin-16C and macrophage migration inhibitory factor from stores in the cytosol
}

\author{
S Roth ${ }^{1}$, M Agthe ${ }^{1}$, S Eickhoff ${ }^{1}$, S Möller ${ }^{1}$, CM Karsten ${ }^{2}$, N Borregaard ${ }^{3}$, W Solbach ${ }^{1}$ and T Laskay ${ }^{1}$
}

Neutrophils harbor a number of preformed effector proteins that allow for immediate antimicrobial functions without the need for time-consuming de novo synthesis. Evidence indicates that neutrophils also contain preformed cytokines, including interleukin (IL)$1 \mathrm{ra}$, CXCL8 and CXCL2. In the search for additional preformed cytokines, a cytokine array analysis identified IL-16 and macrophage migration inhibitory factor (MIF) as preformed cytokines in lysates from human primary neutrophils. Both IL-16 and MIF are unconventional cytokines because they lack a signal sequence. Using confocal immunofluorescence microscopy as well as western blot analysis of subcellular fractions, IL-16 and MIF were found to be stored in the cytosol rather than in the granules of human neutrophils, which implies an unconventional secretion mechanism for both cytokines. IL-16 is synthesized and stored as a precursor (pre-IL-16). We present evidence that the processing of pre-IL-16 to the biologically active IL-16C is mediated by caspase3 and occurs during both spontaneous and UV-induced apoptosis of human neutrophils. Although IL-16 processing occurs during apoptosis, IL-16C and MIF release was observed only during secondary necrosis of neutrophils. Screening a panel of microbial substances and proinflammatory cytokines did not identify a stimulus that induced the release of IL-16C and MIF independent of secondary necrosis. The data presented here suggest that IL-16 and MIF are neutrophil-derived inflammatory mediators released under conditions of insufficient clearance of apoptotic neutrophils, as typically occurs at sites of infection and autoimmunity.

Cell Death Discovery (2015) 1, 15056; doi:10.1038/cddiscovery.2015.56; published online 30 November 2015

\section{INTRODUCTION}

Neutrophil granulocytes have a short lifespan. They undergo apoptosis within a few hours and are cleared from the circulation in the liver, spleen and bone marrow. ${ }^{1}$ At sites of infection and inflammation, their lifespan is prolonged. ${ }^{2}$ However, after having fulfilled their functions, large numbers of neutrophils undergo apoptosis at the site of infection/inflammation.

Apoptotic cell death is generally characterized by chromatin condensation and fragmentation, cell shrinkage, blebbing of the plasma membrane, formation of apoptotic bodies, activation of caspase- 3 and presentation of 'find-me' and 'eat-me' signals. A fundamental feature of apoptotic cell death is the maintenance of membrane integrity in order to prevent leaking of toxic cellular contents. ${ }^{3}$ However, the integrity of the cell membrane of apoptotic neutrophils cannot be maintained for an extended period of time. Consequently, in the case of insufficient clearance, apoptotic neutrophils undergo secondary necrosis. Necrosis is defined by cell lysis, followed by the release of DAMPs (dangerassociated molecular pattern molecules), which results in the activation of inflammatory and immune processes. Whereas primary necrosis is induced by highly toxic substances, leading to the swelling and consequent lysis of cells, secondary necrosis is the consequence of apoptotic cells losing their membrane integrity. Therefore, major differences exist between the release of DAMPs from cells undergoing primary necrosis and that from cells undergoing secondary necrosis. In particular, secondary necrotic cells release considerably less ATP, although they release activated caspase- 3 and proteolytically processed autoantigens. ${ }^{4-6}$

As fast-acting effector cells of the innate immune system, neutrophils are rapidly recruited to sites of infection, where they exert their antimicrobial function. ${ }^{7,8}$ To enable this rapid action, neutrophils harbor preformed antimicrobial effector molecules, such as defensins, lysozyme and cathelicidins, which can act immediately after cell activation without a need for timeconsuming de novo synthesis.,10 Therefore, many of the preformed substances are antimicrobial effector molecules. In addition, neutrophils also contain preformed cytokines, including CXCL8 ${ }^{11}$ and $C X C L 2,{ }^{12}$ which have important roles in the rapid recruitment of inflammatory cells to sites of injury or infection.

In the present study, we searched for additional preformed mediators of inflammation and identified interleukin (IL)-16 and macrophage migration inhibitory factor (MIF) as preformed cytokines in primary human neutrophils. Western blot analysis and confocal microscopy revealed that both IL-16 and MIF are stored in the cytosol rather than in neutrophil granules. We showed that IL-16 is processed in a caspase-3-dependent manner in apoptotic neutrophils, giving rise to the biologically active C-terminal fragment, IL-16C. Importantly, the release of both IL-16 and MIF correlates strongly with the secondary necrosis of neutrophils. We were not able to identify any stimuli that induced the release of IL-16 and MIF independent of neutrophil secondary necrosis. Therefore, IL-16 and MIF represent potential mediators

\footnotetext{
${ }^{1}$ Institute for Medical Microbiology and Hygiene, University of Lübeck, Ratzeburger Allee 160, Lübeck 23562, Germany; ${ }^{2}$ Institute for Systemic Inflammation Research, University of Lübeck, Ratzeburger Allee 160, Lübeck 23562, Germany and ${ }^{3}$ Department of Hematology, Granulocyte Research Laboratory, L-4041, Finsen Center, The National University Hospital, 9 Blegdamsvej, Copenhagen DK-2100, Denmark.

Correspondence: T Laskay (tamas.laskay@uksh.de)
}

Received 1 September 2015; revised 2 October 2015; accepted 16 October 2015; Edited by M Tavassoli 
and modulators of inflammatory and immune responses at sites of insufficient clearance of apoptotic neutrophils.

\section{RESULTS}

IL-16 and MIF are preformed cytokines in primary human neutrophils

Mature neutrophils contain several preformed antimicrobial proteins. ${ }^{13}$ In addition, some cytokines have been shown to be stored in mature neutrophils. ${ }^{11,12,14-20}$ To obtain broader insight into the preformed cytokines of human neutrophils, a lysate from freshly isolated primary human neutrophils was analyzed using the Proteome Profiler Human Cytokine Array Kit (R\&D Systems, Minneapolis, MN, USA). Out of the 36 cytokines, chemokines and acute-phase proteins screened, positive signals were obtained for the cytokines IL-1ra, IL-16, CXCL1 and MIF, indicating the intracellular presence of these cytokines in resting human neutrophils (Figure 1). In addition, a signal for sICAM-1 was detected (Figure 1). However, because a cell lysate rather than cell culture supernatant was analyzed with the Cytokine Array Kit in our study, the sICAM-1 signal likely indicates the presence of ICAM-1 in the neutrophil cell membrane rather than the soluble form of ICAM-1.

Preformed IL-16 and MIF are localized in the cytosol of human neutrophils

Antimicrobial proteins represent the majority of preformed proteins in neutrophils. They are formed during granulopoiesis and stored in neutrophil granules. ${ }^{21}$ Neutrophils possess at least four different types of granules: (i) azurophilic/primary granules, (ii) specific/secondary granules, (iii) gelatinase/tertiary granules and (iv) secretory vesicles. The various granule populations contain distinct sets of proteins, some of which are used as identification markers for the particular granule type.

IL-16 and MIF share a feature that is rather uncommon for cytokines: they both lack a signal sequence. ${ }^{2,23}$ Having observed that these two cytokines with this unusual feature are present in neutrophils, we addressed the issue of the subcellular localization and release of IL-16 and MIF by primary human neutrophils in vitro by applying immunofluorescence staining and confocal fluorescence microscopy. Imaging confirmed the intracellular presence of preformed IL-16 and MIF in neutrophils (Figure 2a). The white spots/areas shown represent the results of the computer-assisted analysis of colocalization of the green IL-16 signals with the red signals of S100A8 (Figure 2a, III and IV). The analysis showed a strong colocalization of IL-16 with S100A8, an abundant cytosolic protein in neutrophils (Figure $2 \mathrm{a}$, IV). In contrast, colocalization analysis of IL-16 with gelatinase (MMP9; Figure 2a, I and II), a protein contained in both gelatinase and specific granules, showed a weak colocalization with IL-16. Similarly, a very strong colocalization between MIF and S100A8 signals was also observed (Figure 2a, VII and VIII), whereas only very weak colocalization was observed between the MIF and MMP9 signals (Figure 2a, V and VI).

\begin{tabular}{|lllll|}
\hline pos & & CXCL1 & sICAM-1 & pos \\
& IL-1ra & & & \\
pos & & IL-16 & & \\
\hline
\end{tabular}

Figure 1. Preformed cytokines in human neutrophils. Lysate of freshly isolated neutrophils was analyzed for preformed cytokines using Human Cytokine Array Panel A, screening 36 cytokines, chemokines and acute-phase proteins. pos, positive control.
The data thus indicate that IL-16 and MIF are stored in the cytosol rather than in the granules of neutrophils. To confirm this finding, subcellular fractions of neutrophils were analyzed using western blot analysis. The total cell homogenate as well as five subcellular fractions, that is, azurophilic granules, specific granules, gelatinase granules, secretory vesicles/plasma membrane and cytosol, were assessed for the presence of IL-16 and MIF. This analysis showed that IL-16 and MIF are present in the cytosol rather than in any of the granules or vesicles (Figure $2 b$ ). Therefore, this analysis clearly confirms the intracellular presence and cytosolic localization of both IL-16 and MIF in primary human neutrophils.

pre-IL-16 is processed in apoptotic neutrophils in a caspase-3-dependent manner

IL-16 is synthesized as an $\sim 80-k D a$ precursor molecule, ${ }^{24}$ recently termed pre-IL-16. ${ }^{25}$ Previously, it was shown in $\mathrm{CD}^{+}$and $\mathrm{CD} 8^{+}$ $\mathrm{T}$ cells that pre-IL-16 is cleaved enzymatically by caspase- $3,{ }^{23,26}$ a cysteine-aspartic acid protease that has a major role in apoptosis. Caspase-3-mediated processing of pre-IL-16 results in the generation of the $17-\mathrm{kDa}$ peptide IL-16C and the larger fragment pro$\mathrm{IL}-16 .^{23} \mathrm{IL}-16 \mathrm{C}$ is the secreted fragment, exerting chemotactic activity on $\mathrm{CD}^{+}$cells. $^{27,28}$

Neutrophils undergo spontaneous apoptosis within $40 \mathrm{~h}$ when cultured in vitro. ${ }^{29}$ To investigate whether pre-IL-16 is cleaved into the biologically active IL-16C during neutrophil apoptosis, neutrophils were analyzed after 5, 10, 20, 30, 40 and $60 \mathrm{~h}$ of incubation in vitro. The proportions of apoptotic cells were assessed by staining cells with Annexin-V-FLUOS and propidium iodide (PI). The intracellular levels of pre-IL-16 and IL-16C were assessed using western blot analysis.

Analysis of freshly isolated neutrophils revealed a double band at $75-80 \mathrm{kDa}$ and a single band at $55 \mathrm{kDa}$ (Figure $3 \mathrm{a}$, Supplementary Information and Supplementary Figure 1). The intensities of all three bands decreased with increasing incubation times. After $10 \mathrm{~h}$ of incubation, the 17-kDa IL-16C band appeared (Figure 3a). The intensity of the IL-16C band became stronger with an increased duration of incubation. A strong negative correlation between the abundance of the non-processed pre-IL-16 band and apoptosis became evident (Figure $3 \mathrm{~b}$, left panel). At the same time, a strong correlation was observed between the level of the IL-16C band and neutrophil apoptosis (Figure 3b, right panel). These results indicate that pre-IL-16 is indeed processed during apoptotic cell death in neutrophils.

Our results suggest that pre-IL-16 is cleaved by caspase- 3 , as has been observed in T cells. ${ }^{26}$ To confirm this hypothesis, neutrophil apoptosis was induced by irradiation with $200 \mathrm{~mJ} / \mathrm{cm}^{2}$ UV light $(256 \mathrm{~nm})$. Immediately after irradiation, neutrophils were treated with a pan-caspase inhibitor (Q-VD-OPh), a caspase-3-specific inhibitor (Z-DEVD-FMK) and the solvent DMSO as control. After $6 \mathrm{~h}$ of incubation at $37^{\circ} \mathrm{C}$, neutrophil viability was assessed with Annexin-V-FLUOS and PI staining. The intracellular levels of preIL-16 and IL-16C were assessed with western blot analysis.

UV light irradiation increased the apoptosis from 20 to 92\%, inducing caspase- 3 activation and pre-IL-16 processing into IL-16C after $6 \mathrm{~h}$ of incubation (Figure 3c). Treatment with Q-VD-OPh and Z-DEVD-FMK significantly inhibited both apoptosis and the processing of pre-IL-16 into IL-16C by blocking the autocatalytic cleavage of the p20 fragment of caspase- 3 into the $p 17$ fragment (Figure 3c). Processing and consequent activation of caspase- 3 occur in a two-step process. ${ }^{30}$ First, pro-caspase-3 is cleaved by the upstream caspases- 8 and -9 into a heterotetramer complex consisting of two p20 and two p12 fragments. In the second step, the p20 fragments are autocatalytically processed into p17 fragments, forming the mature and biologically active p17/p12-caspase-3 complex. Our results show that it is the p17/p12-caspase-3 complex that mediates IL-16 processing in neutrophils. 
a
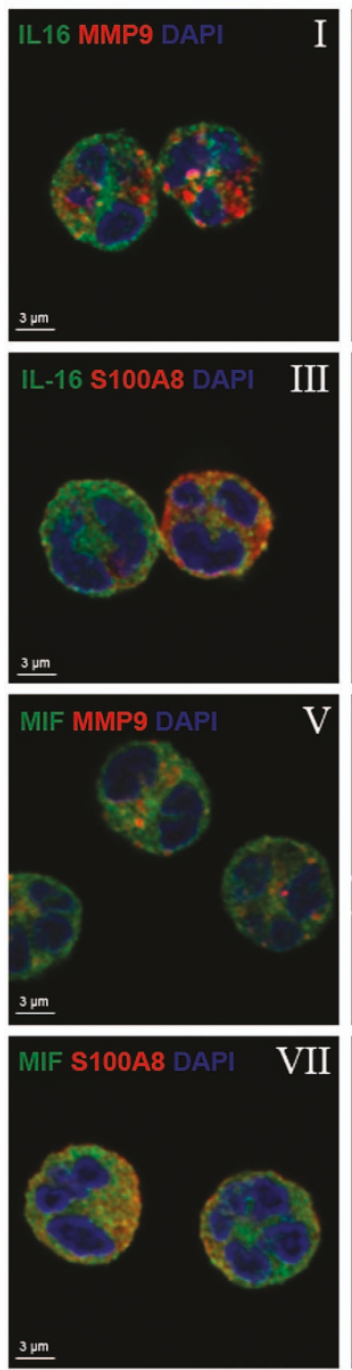
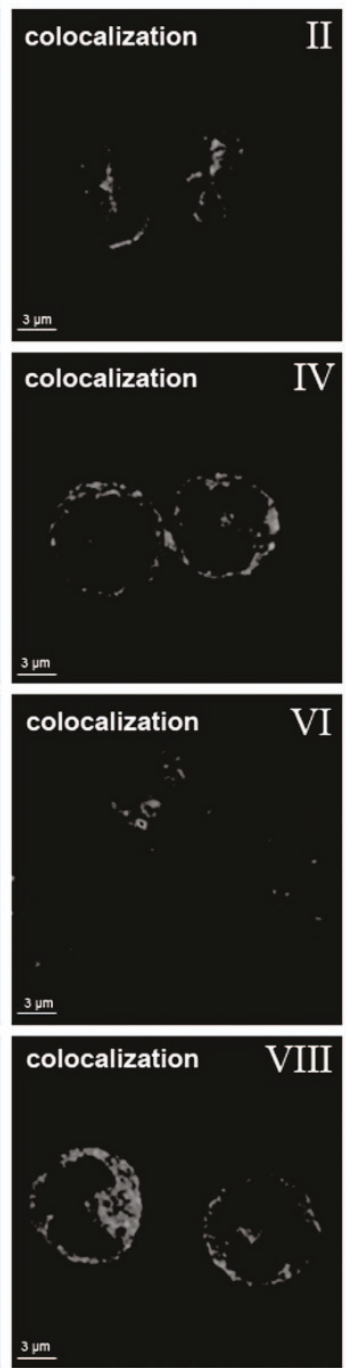

b

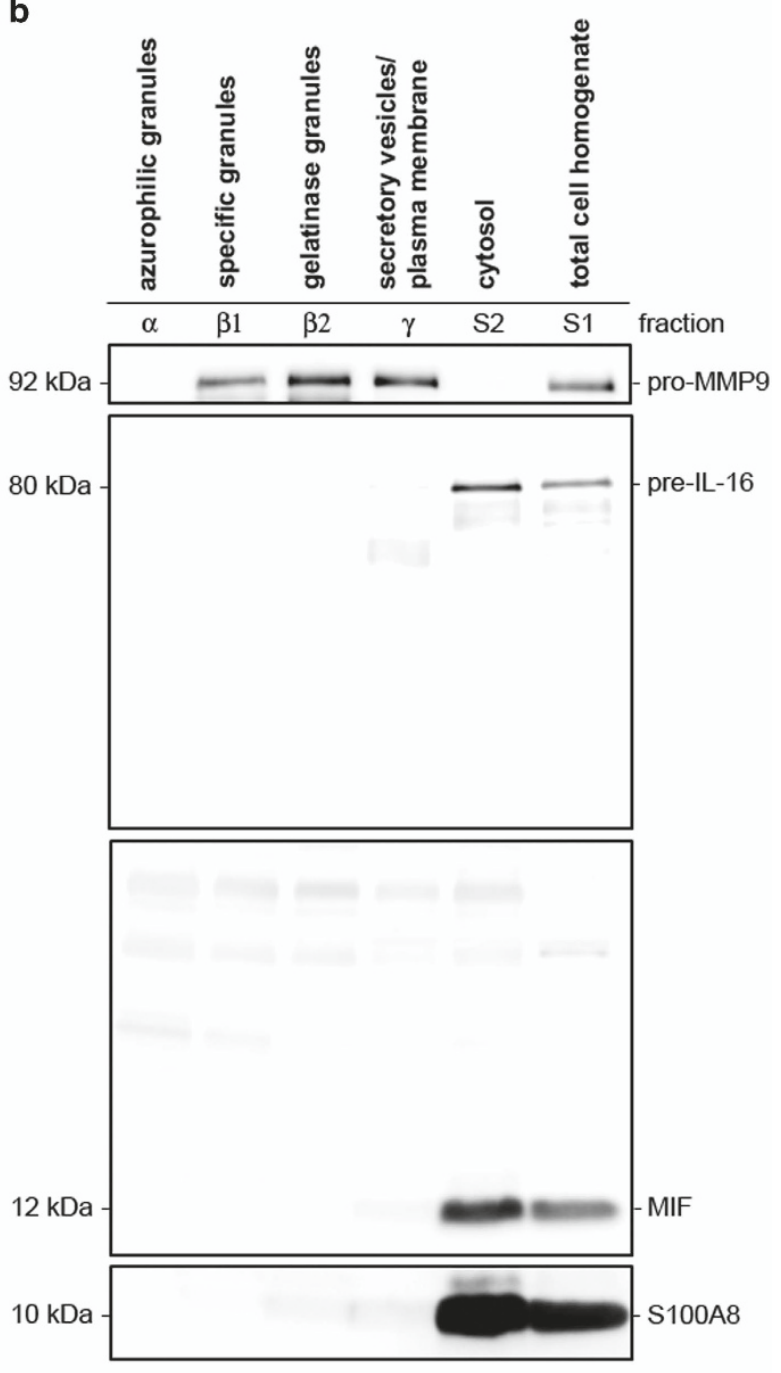

Figure 2. Cytosolic storage of IL-16 and MIF in neutrophils. (a) Neutrophils were fixed and stained for IL-16 (green, I and III), MIF (green, V and VII), MMP9 (red, I and V), S100A8 (red, III and VII) and the nuclei (DAPI, blue; I, III, V and VII). Staining was analyzed using confocal microscopy. Colocalization studies of the fluorescent signals for IL-16 and MMP9 (II), IL-16 and S100A8 (IV), MIF and MMP9 (VI), MIF and S100A8 (VIII) were performed. White signals indicate colocalization of the green and red fluorescent signals. Bars, $3 \mu \mathrm{m}$. The presented data are representative of three independent experiments. (b) Freshly isolated neutrophils were fractionated in a two-step Percoll gradient and analyzed for its content of IL-16, MIF, pro-MMP9 and S100A8 using western blot analysis. The presented data are representative of two experiments.

IL-16C and MIF are released from neutrophils during secondary necrosis

Because IL-16 is processed during neutrophil apoptosis (Figure 3), we addressed the question of whether the IL-16C peptide was secreted by apoptotic neutrophils. Supernatants of in vitro cultured neutrophils were collected at various time points during $60 \mathrm{~h}$ of incubation, ultracentrifuged and their content of IL-16 and MIF determined. The proportion of apoptotic and necrotic cells at the indicated time points was assessed using flow cytometry following staining with Annexin-V-FLUOS and PI. In addition, the lactate dehydrogenase (LDH) enzyme activity was detected in the cell culture supernatants as a measure of cell death-associated release of cellular content. As early as $5 \mathrm{~h}$ of incubation, $\sim 10 \%$ of the cells were apoptotic; this proportion increased to $\sim 35 \%$ after $10 \mathrm{~h}$ and to more than $60 \%$ after $20 \mathrm{~h}$ of incubation (Figure $4 \mathrm{a}$ ). Only a small proportion of necrotic cells was detected $20 \mathrm{~h}$ after incubation. After that, the proportion of necrotic cells increased gradually during the $60 \mathrm{~h}$ of culture (Figure 4a). Similarly, LDH release was first detected after $20 \mathrm{~h}$ of culture. After this time point, the LDH levels of the culture supernatants increased with time, indicating enhanced cell death during prolonged in vitro culture (Figure $4 a$, top). Notably, IL-16 release did not have the same kinetics as apoptosis. Instead, IL-16 could be detected in the supernatants only after the appearance of secondary necrotic cells (Figure 4a). A very strong correlation was observed between IL-16 levels and the percentage of secondary necrotic cells as well as $\mathrm{LDH}$ release (Figures $4 \mathrm{c}$ and $\mathrm{d}$, top). However, the correlation between IL-16 release and the proportion of apoptosis was notably weaker and not significant (Figure $4 b$, top).

Biologically active MIF does not require caspase-dependent proteolytic processing. However, in a previous study, MIF release was observed from apoptotic but not from viable neutrophils. ${ }^{14}$ We assessed the release of MIF from neutrophils at various time points during the $60 \mathrm{~h}$ of in vitro culture. MIF release did not follow the kinetics of apoptosis but rather the kinetics of secondary necrosis of neutrophils (Figure 4a). MIF appeared in the supernatants only when necrotic cells and LDH release were detectable. Similar to IL-16, a very strong and significant correlation was 

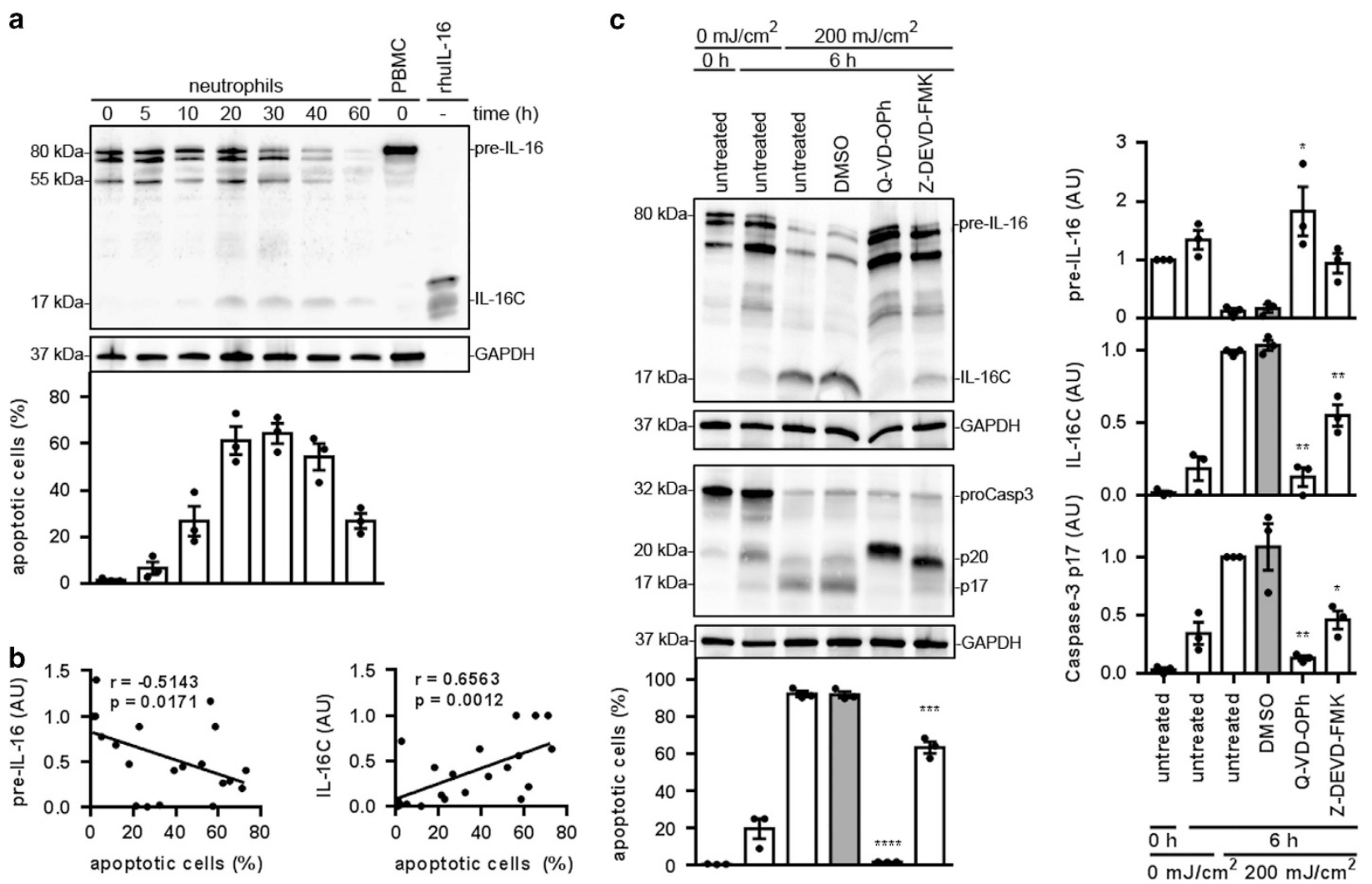

Figure 3. Caspase-3-mediated processing of pre-IL-16 in apoptotic neutrophils. (a and b) Neutrophils were cultured for 0, 5, 10, 20, 30, 40 and $60 \mathrm{~h}$ at $37^{\circ} \mathrm{C}$. (a) Lysates were prepared at the indicated time points and analyzed for IL-16 and GAPDH (loading control) using western blot analysis. Shown are representative blots of three independent experiments. Neutrophil viability was assessed with flow cytometry using Annexin-V-FLUOS and PI staining. Bars represent the mean of the proportion of apoptotic cells (Annexin-V-FLUOS $\left.{ }^{+} / \mathrm{PI}^{-}\right) \pm$S.E.M. of three independent experiments. Individual data points are shown as black dots. (b) The correlation between pre-IL-16 (left)/IL-16C (right) levels (normalized to GAPDH) and the proportion of apoptotic cells. The data were analyzed by the nonparametric Spearman correlation, $n=21$, $P$ (two-tailed). (c) Neutrophils were irradiated with $200 \mathrm{~mJ} / \mathrm{cm}^{2}$ UV light $(256 \mathrm{~nm})$. Immediately after radiation, the neutrophils were treated with the pan-caspase inhibitor (Q-VD-OPh, $1 \mu \mathrm{M}$ ), the caspase-3-specific inhibitor (Z-DEVD-FMK, $100 \mu \mathrm{M})$ and the solvent DMSO (1:200). Lysates were prepared after $6 \mathrm{~h}$ of incubation at $37^{\circ} \mathrm{C}$ and analyzed for IL-16, caspase-3 and GAPDH (loading control) using western blot analysis. Shown are representative blots of three independent experiments (left panel), which were quantified and normalized to GAPDH (right panel). Neutrophil viability was assessed by flow cytometry using Annexin V-FLUOS and PI staining. Bars represent the mean of the percentage of apoptotic cells (Annexin V-FLUOS $\left.{ }^{+} / \mathrm{PI}^{-}\right) \pm$S.E.M. of three independent experiments $\left({ }^{*} P<0.05 ;{ }^{* *} P<0.01\right.$; ${ }^{* * *} P<0.001$; ${ }^{* * * *} P<0.0001$ compared with the DMSO sample by one-way ANOVA followed by Holm Sidak multiple comparison correction). Individual data points are shown as black dots. AU, arbitrary units.

observed between the proportion of secondary necrotic cells and MIF release as well as between $\mathrm{LDH}$ release and MIF release (Figures $4 \mathrm{c}$ and $\mathrm{d}$, bottom). Only a weak and nonsignificant correlation was observed between MIF release and the proportion of apoptosis (Figure 4b, bottom).

Our results revealed that IL-16C and MIF are released from secondary necrotic rather than apoptotic neutrophils. In an attempt to clarify the role of apoptosis in the release of IL-16C and MIF, we modulated neutrophil apoptosis. For this purpose, spontaneous neutrophil apoptosis was facilitated using UV-light irradiation $(256 \mathrm{~nm})$. UV-light irradiation enhanced neutrophil apoptosis in a dose-dependent manner (Figure 5a). Importantly, although irradiation with $200 \mathrm{~mJ} / \mathrm{cm}^{2}$ UV-light-induced apoptosis in $\sim 80 \%$ of neutrophils, no significant release of IL-16 and MIF was observed. However, irradiation with higher UV doses led to the release of high IL-16 and MIF levels (Figure 5a). Notably, increasing the UV dose enhanced the degree of apoptosis only marginally but led to the induction of secondary necrosis in $\sim 25 \%$ of neutrophils (Figure 5a). These results demonstrate once more that IL-16C and MIF are released not from apoptotic neutrophils but from secondary necrotic neutrophils, as has been shown during spontaneous cell death.
As another approach for modulating neutrophil apoptosis, neutrophils were infected with Anaplasma phagocytophilum, an intracellular bacterium that preferentially infects neutrophil granulocytes. Infection with A. phagocytophilum has been shown to inhibit neutrophil apoptosis. ${ }^{31}$ As expected, after $6 \mathrm{~h}$ of incubation, neutrophils in the infected culture had a lower level of apoptosis (Figure 5b). Still, cells in the infected culture released significantly higher levels of IL-16 and apparently higher levels of MIF compared with uninfected neutrophils (Figure 5b). However, infected cells also contained a significantly higher proportion of necrotic neutrophils (Figure $5 b$ ), substantiating the view that the release of IL-16 and MIF is dependent on secondary necrosis rather than apoptosis.

\section{DISCUSSION}

Neutrophils are capable of expressing and secreting more than 50 different cytokines. ${ }^{32} \mathrm{~A}$ few of these, such as IL-1 $\mathrm{ra}^{20} \mathrm{IL}-4,{ }^{17} \mathrm{IL}-6,{ }^{15}$ CXCL8, ${ }^{11}$ IL-12, ${ }^{16} \mathrm{MIF}^{14}$ CXCL2, ${ }^{12}$ TGF- $a^{19}$ and VEGF, ${ }^{33}$ are already produced during granulopoiesis and form pools of preformed cytokines in mature neutrophils. As the secretion of preformed cytokines is not delayed by prior de novo synthesis, these 


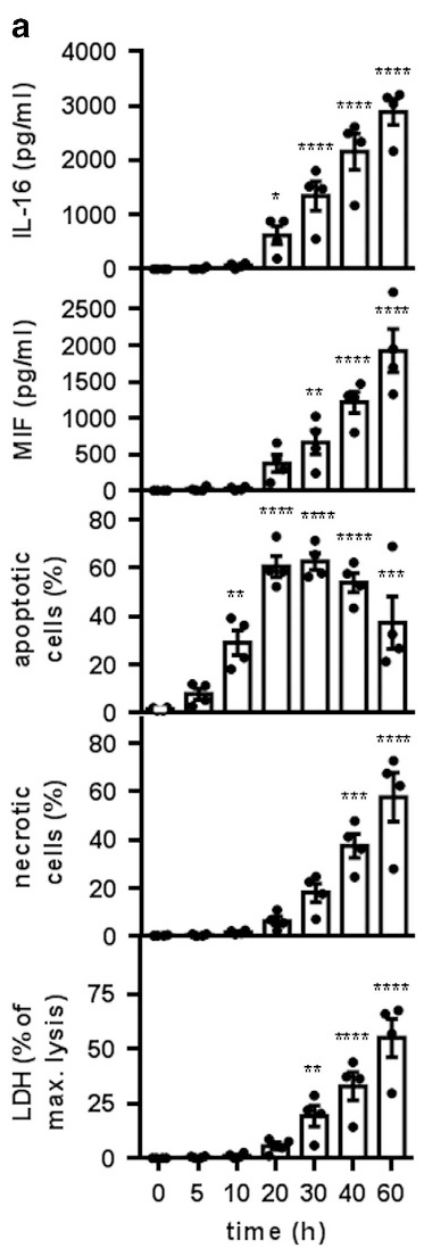

b
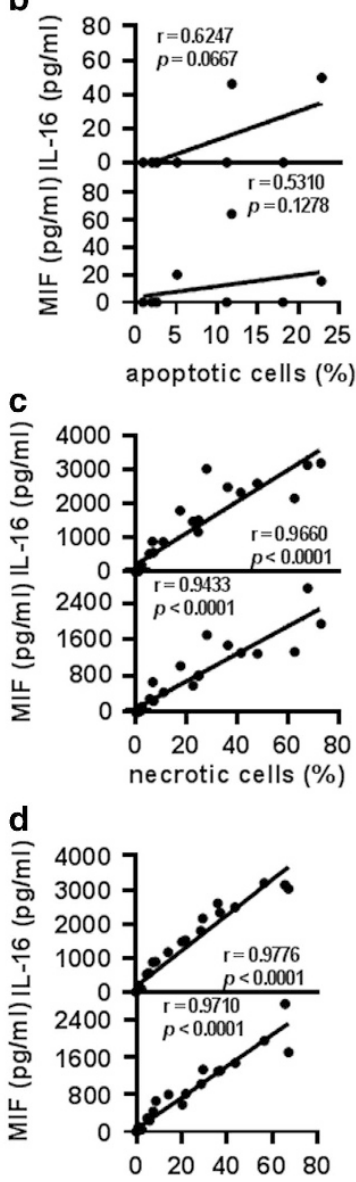

LDH (\% of max. lysis)
Figure 4. IL-16C release during secondary necrosis of neutrophils. (a-d) Neutrophils were incubated for $0,5,10,20,30,40$ and $60 \mathrm{~h}$ at $37^{\circ} \mathrm{C}$. (a) At the indicated time points, the samples were centrifuged at $400 \times g$ for $10 \mathrm{~min}$. Supernatants were collected and centrifuged at $4{ }^{\circ} \mathrm{C}$ and $60000 \times g$ for $1 \mathrm{~h}$ before being analyzed with ELISA for released IL-16 and MIF and with a cytotoxicity detection kit for LDH (depicted in \% of max. lysis, as obtained by lysis of freshly isolated neutrophils with Triton X-100). Neutrophil viability was assessed with flow cytometry using Annexin-V-FLUOS and PI staining. Bars represent the means \pm S.E.M. of four independent experiments $\left({ }^{*} P<0.05 ;{ }^{* *} P<0.01 ;{ }^{* * *} P<0.001,{ }^{* * * *} P<0.0001\right.$ compared with the 0 -h sample by one-way ANOVA followed by Holm Sidak multiple comparisons correction). Individual data points are shown as black dots. (b-d) Correlation analysis of the data obtained in $\mathbf{a}$, analyzed by nonparametric Spearman correlation, $P$ (two-tailed). (b) The correlation between the percentage of apoptotic neutrophils and the concentration of IL-16 (top) or MIF (bottom) in the supernatants. Samples containing more than $3 \%$ necrotic cells were excluded. $n=10$. (c) The correlation between the percentage of necrotic neutrophils and the concentration of IL-16 (top) or MIF (bottom) in the supernatants. $n=28$. (d) The correlation between the percentage of $\mathrm{LDH}$ release and the concentration of IL-16 (top) or MIF (bottom) in the neutrophil supernatants. $n=28$.

cytokines are believed to have a specific role during the early phase of inflammation.

Using two gradient centrifugations and endotoxin-free reagents, we isolated neutrophils with minimal or no activation and contamination with other immune cells. In a lysate from these neutrophils, we identified the presence of CXCL1, IL-1 ra, IL-16, MIF and SICAM- 1 among the 36 cytokines and acute-phase proteins screened. This is in accordance with previous studies that also found IL-1 $\mathrm{ra}^{20}$ and MIF ${ }^{14}$ to be preformed cytokines in human
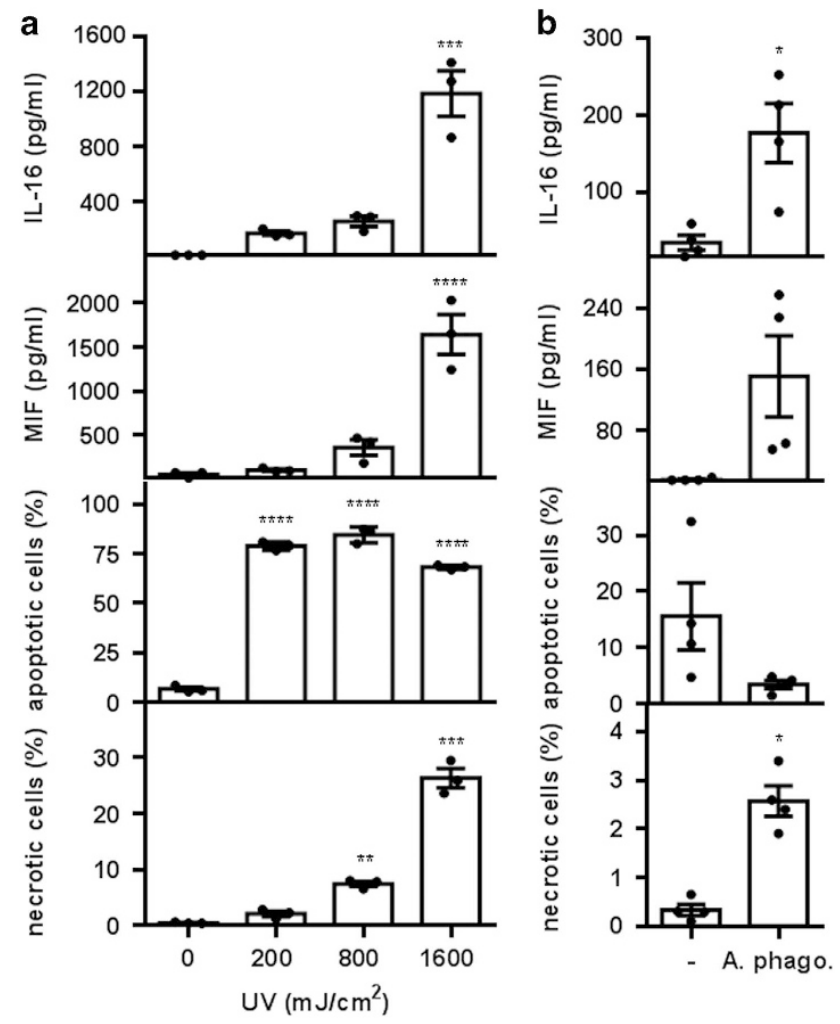

Figure 5. IL-16C release upon treatments inducing secondary necrosis of neutrophils. (a) Neutrophils were irradiated with 0, 200, 800 and $1600 \mathrm{~mJ} / \mathrm{cm}^{2}$ UV light $(256 \mathrm{~nm})$. After $6 \mathrm{~h}$ of incubation at $37^{\circ} \mathrm{C}$, the supernatants were analyzed with ELISA for released IL-16 and MIF. Neutrophil viability was assessed with flow cytometry using Annexin-V-FLUOS and PI staining. Bars represent the means \pm S.E.M. of three independent experiments $\left({ }^{*} P<0.05 ;{ }^{*} P<0.01 ;{ }^{* *} P<0.001\right.$, ${ }^{* * * *} P<0.0001$ compared with the 0 -h sample by one-way ANOVA followed by Holm Sidak multiple comparison correction). Individual data points are shown as black dots. (b) Neutrophils were infected with A. phagocytophilum (A. phago) for 6 at $37^{\circ} \mathrm{C}$. The supernatants were analyzed with ELISA for released IL-16 and MIF, and neutrophil viability was assessed with flow cytometry using Annexin-V-FLUOS and $\mathrm{PI}$ staining. Bars represent the means \pm S.E.M. of four independent experiments $\left({ }^{*} P<0.05{ }^{* * *} P<0.01{ }^{* * *} P<0.001\right.$, ****P $<0.0001$ compared with the uninfected control by paired $t$-test). Individual data points are shown as black dots.

neutrophils. Moreover, CXCL2 (MIP-2), the murine homolog of CXCL1 and CXCL8, has been described to be a preformed cytokine in murine neutrophils. ${ }^{12}$ The results of our array now confirm that CXCL1 is also preformed in human neutrophils. In contrast to this finding, our screening did not detect IL-6 and IL-12 among the preformed cytokines in human neutrophils, although the preformed presence of these cytokines has been described in murine neutrophils. ${ }^{15,16}$ Furthermore, we could not confirm the intracellular storage of IL- $4{ }^{17}$ and $\mathrm{CXCL} 8,{ }^{11}$ as has been shown previously in human neutrophils. However, as these stores are supposed to contain only small amounts of IL-4 and CXCL8, the sensitivity of our array may not have been sufficient to detect these cytokines.

Although the presence of preformed IL-1 $\mathrm{ra}^{20}$ and $\mathrm{MIF}^{14}$ has been described before in human neutrophils, IL-16 has not. There has even been doubt raised as to whether neutrophils express IL-16 at all. ${ }^{32}$ In other cells, IL-16 is synthesized as a precursor molecule (pre-IL-16), which is cleaved by caspase-3 into the secreted and biologically active IL-16C. ${ }^{23,24,26}$ If pre-IL-16 is a preformed cytokine in human neutrophils, it should also be processed during apoptosis. Indeed, our western blot analysis 

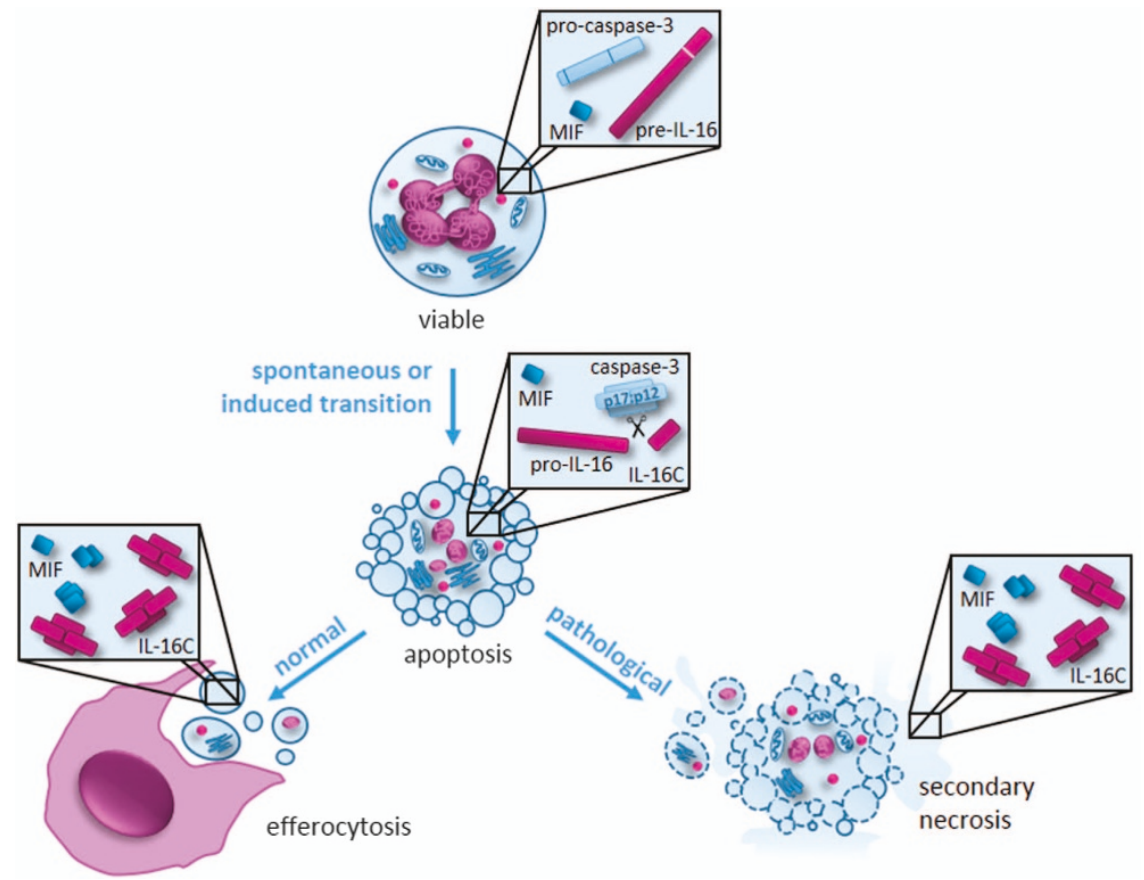

Figure 6. Schematic representation of the storage, processing and release of IL-16 and MIF in human neutrophils. MIF and IL-16 are preformed cytokines stored in the cytosol of human neutrophils. During apoptosis, the precursor of IL-16 (pre-IL-16) is processed into the biologically active IL-16C in a caspase-3-dependent manner. While under normal conditions, apoptotic cells are cleared by phagocytes, this process is impaired in infections and autoimmunity, leading to the release of MIF and IL-16C by secondary necrotic neutrophils.

showed that pre-IL-16 is cleaved into IL-16C during apoptosis of human neutrophils. Because caspase-3 inhibitors block pre-IL-16 cleavage in human neutrophils, the processing of pre-IL-16 is dependent on caspase-3 activation and presumably does not involve other proteases. Nonetheless, it has been shown in T cells that caspase-3 activation and pre-IL-16 processing are not automatically accompanied by apoptosis. We are not aware of apoptosis-independent activation of caspase-3 in neutrophils, nor were we able to detect a constant background caspase-3 activation, as postulated for $\mathrm{CD} 8^{+} \mathrm{T}_{\text {cells. }}{ }^{26}$ Therefore, we assume that apoptosis, caspase- 3 activation and pre-IL-16 processing are joint processes in human neutrophils.

Notably, in other studies, caspase-3 activation in the context of IL-16 processing was assessed by the appearance of the p20 fragment only. The p20 fragment of caspase- 3 is a subunit of the p20:p12 heterodimer, which in a second step is autocatalytically cleaved into the p17 fragment, which forms the active p17:p12 caspase- 3 heterodimer. ${ }^{30}$ However, recent publications also indicate a biological function of the intermediate heterodimer p20:p12, which is able to activate PKC $\delta$, which then mediates the NFKB-dependent synthesis of proinflammatory cytokines. ${ }^{34}$ Our data suggest that pre-IL-16 in neutrophils is cleaved by the apoptosis-associated p17:p12 heterodimer rather than the inflammation-associated p20:p12 heterodimer of caspase-3.

Most of the preformed proteins of neutrophils are packaged into granules during granulopoiesis. However, this process is questionable for IL-16 and MIF. Both cytokines lack signal peptides and therefore do not enter the ER and the Golgi apparatus. Consequently, they do not follow the conventional secretory pathway. Examining the intracellular localization of IL-16 and MIF, we found both cytokines to be localized in the cytosol of neutrophils, along with the calcium-binding S100A8 protein. ${ }^{35}$ This finding implies that IL-16 and MIF need to use unconventional pathways to be released, as described already for IL-1 $\beta^{36}$ and FGF2. ${ }^{37}$ Testing a panel of cytokines, microbial constituents and glucocorticoids in vitro, we were not able find a stimulus that induced an active release of IL-16 and MIF by neutrophils (data not shown). Our data suggest that IL-16C and MIF are released passively by secondary necrotic neutrophils rather than actively by apoptotic neutrophils. As necrostatin-1, an inhibitor of necroptosis, did not affect the ratio of apoptotic and necrotic cells during a 72-h in vitro culture (data not shown), necroptosis is unlikely to have a role in spontaneous cell death of neutrophils. Accordingly, necrostatin-1 did not inhibit IL-16 release of neutrophils (data not shown). The predication that IL-16C and MIF are released by secondary necrotic neutrophils is strengthened by our results that after UV light irradiation and infection with A. phagocytophilum, IL-16 and MIF release is in line with enhanced secondary necrosis, but not with apoptosis. This result contradicts the finding of Daryadel et al., ${ }^{14}$ who showed that MIF is released by apoptotic neutrophils dependent on $A B C$ transporters, indicating an active release. However, other studies also describe the active secretion of IL-16 and MIF after S100A8, ${ }^{38} \mathrm{GM}^{-\mathrm{CSF}^{38}}$ and $\mathrm{C}^{3} \mathrm{a}^{39}$ treatment. Repeating their protocols, we did not observe an elevated IL-16 and MIF release after treatment of our neutrophil preparations with S100A8, GM-CSF or C5a. On the contrary, as C5a and GM-CSF treatment decreased apoptosis and secondary necrosis after $24 \mathrm{~h}$ of incubation, we detected even less IL-16 and MIF in the supernatants of treated neutrophils (data not shown). Moreover, in studies by Riedemann et al. ${ }^{40}$ and Daryadel et al. ${ }^{14}$ the MIF concentrations in the supernatants of neutrophils are much higher than in our samples, reaching up to $8 \mathrm{ng} / \mathrm{ml}$ after $6 \mathrm{~h}$ of incubation of $5 \times 10^{6}$ cells. Because the MIF concentrations measured by us using similar settings were always less than $50 \mathrm{pg} / \mathrm{ml}$, we believe that the high concentrations in the other studies may be because of another isolation protocol for neutrophils, leading to more contamination with other immune cells.

As glucocorticoids are known to be potent inducers of MIF secretion from monocytes, ${ }^{41}$ we treated neutrophils with $1 \mu \mathrm{M}-0.1 \mathrm{fM}$ dexamethasone or hydrocortisone for $12 \mathrm{~h}$. As described previously, we observed an anti-apoptotic effect ${ }^{42}$ as well as a concomitant decrease in IL-16 and MIF release in the cells treated with higher concentrations of both glucocorticoids (data not shown). 
In our hands, treatment with commonly used pro- and antiinflammatory substances did not induce an active release of IL-16 and MIF by neutrophils; hence, the question arises of why these two cytokines exist as preformed cytokines when they are possibly only released during secondary necrosis. Although in the healthy organism apoptotic cells are immediately efferocytosed by professional phagocytes, this may not be the case during microbial and viral infections, as well as in autoimmune diseases. In this context, infection with HIV provides an interesting example. As $\mathrm{H}_{2} \mathrm{O}_{2}$ produced by neutrophils is viricidal to $\mathrm{HIV}-1,{ }^{43}$ the virus impairs the function of $\mathrm{H}_{2} \mathrm{O}_{2}$ release and accelerates the apoptosis of neutrophils. ${ }^{44}$ Moreover, the HIV protein Nef inhibits the efferocytosis of apoptotic neutrophils by macrophages and monocytes. ${ }^{45}$ Ineffective clearance of apoptotic neutrophils will cause the transition to secondary necrosis. As IL-16C was shown to inhibit the replication of HIV-1, ${ }^{46,47}$ neutrophils may have found another way to reduce virus propagation by processing pre-IL-16 into IL-16C during apoptosis and releasing IL-16C during secondary necrosis. Moreover, impaired efferocytosis, ${ }^{48,49}$ as well as elevated expression levels of IL-16 $6^{50,51}$ and MIF, ${ }^{52,53}$ have been observed to be connected with increased disease severity in autoimmune diseases such as rheumatoid arthritis and systemic lupus erythematosus. Although MIF has been shown to have a critical role in recruiting inflammatory immune cells in diseases such as rheumatoid arthritis, ${ }^{54,55}$ the biological role of IL-16 in autoimmune diseases still needs to be unraveled.

In conclusion, the present study demonstrates that IL-16 and MIF are preformed cytokines present in the cytosol of mature human neutrophils that are released exclusively during secondary necrosis (Figure 6). Thus, it is plausible to assume that IL-16 and MIF contribute pivotally to the inflammatory immune reactions observed in diseases associated with defective clearance of senescent cells.

\section{MATERIALS AND METHODS}

Isolation and culture of human neutrophils

Heparinized peripheral blood was obtained from healthy volunteers with written informed consent. All studies were approved by the ethics committee of the University of Lübeck (05-124). Granulocytes were isolated as described previously using discontinuous Percoll gradient centrifugation. ${ }^{56}$ Granulocyte purity and viability were $>99.9 \%$, as assessed by differential staining with Diff-Quik (Medion Diagnostics, Düdingen, Switzerland) and Annexin-V-FLUOS/PI staining. Only preparations with $<3 \%$ eosinophils were used for the experiments.

All experiments were conducted using complete medium (RPMI 1640 medium (Sigma-Aldrich, Steinheim, Germany) supplemented with $10 \%$ FCS (Sigma-Aldrich), $50 \mu \mathrm{M}$ 2-mercaptoethanol (Sigma-Aldrich), $4 \mathrm{mM}$ L-glutamine (Biochrom, Berlin, Germany), $10 \mathrm{mM}$ HEPES (Gibco Life Technologies, Paisley, Great Britain), $100 \mathrm{U} / \mathrm{ml}$ penicillin and $100 \mu \mathrm{g} / \mathrm{ml}$ streptomycin (Biochrom)).

\section{Cytokine array}

Freshly isolated neutrophils $\left(1.5 \times 10^{7}\right)$ were resuspended in lysing buffer (1\% Triton X-100 (Merck Millipore, Darmstadt, Germany), $10 \mu \mathrm{g} / \mathrm{ml}$ aprotinin (Sigma-Aldrich), $10 \mu \mathrm{g} / \mathrm{ml}$ leupeptin (Sigma-Aldrich) and $10 \mu \mathrm{g} / \mathrm{ml}$ pepstatin A (Sigma-Aldrich) in PBS). After additional $-70^{\circ} \mathrm{C}$ freeze-and-thaw-disruption, the sample was centrifuged at $10000 \times g$ for $5 \mathrm{~min}$. The supernatants were collected and tested for preformed cytokines using the Proteome Profiler Array-Human Cytokine Array Panel A (R\&D Systems) according to the manufacturer's instructions.

\section{Immunofluorescence staining}

Freshly isolated neutrophils were fixed (4\% PFA), permeabilized $(0.1 \%$ Triton X-100 in PBS) and blocked (10\% BSA, 10\% donkey serum). Human IL-16 C-terminal peptide antibody (Ab; R\&D Systems, $1: 25$ ), human MIF Ab (R\&D Systems, $1: 25$ ), anti-MRP Ab (Abcam, Cambridge, UK, $1: 200$ ) and anti-MMP9 Ab (Abcam, 1:50) were added and the cells were incubated at $4{ }^{\circ} \mathrm{C}$ for $12 \mathrm{~h}$. The cells were washed and incubated with donkey anti-goat
AlexaFluor 488 (Molecular Probes, Eugene, OR, USA, $1: 200$ ) for $1 \mathrm{~h}$ at room temperature. After washing and blocking (10\% BSA, 10\% goat serum), the cells were incubated with goat anti-rabbit AlexaFluor 555 (Cell Signaling Technologies, Danvers, MA, USA, 1:500) for $1 \mathrm{~h}$ at room temperature before being washed again. The cells were then mounted with ProLong Gold antifade reagent containing DAPI (Molecular Probes, Grand Island, NY, USA). Neutrophils stained only with the secondary Ab were used as a negative control. Images were obtained using an Olympus FV1000 confocal microscope (Olympus, Center Valley, PA, USA) with a $\times 60$, $\mathrm{NA}=1.35$ oil objective. Image capturing and analysis were performed using the Fluoview 2.1c software (Olympus). For colocalization studies, data sets were analyzed using the IMARIS software (Bitplane Scientific Software, South Windsor, CT, USA).

\section{Preparation of neutrophilic subcellular fractions}

Granule subsets of neutrophils were purified applying a three-layer Percoll density gradient, as specified by Kjeldsen et al. ${ }^{57}$ Aliquots of each fraction were assayed for the presence of marker proteins by ELISA. ${ }^{58}$ The fractions were pooled as follows: $1-7$ were the $\alpha$-band, $8-13$ the $\beta 1$-band, $14-17$ the $\beta 2$-band, 18-27 the $\gamma$-band and 28-39 the cytosol.

\section{Annexin-V-FLUOS/PI assay}

For the Annexin-V-FLUOS/PI staining, cells were double-stained with Annexin-V-FLUOS (Roche, Mannheim, Germany) and PI (Sigma-Aldrich) according to the manufacturer's instructions. The percentages of viable (Annexin-V-FLUOS ${ }^{-} / \mathrm{PI}^{-}$), apoptotic (Annexin-V-FLUOS ${ }^{+} / \mathrm{PI}^{-}$) and necrotic cells (Annexin-V-FLUOS ${ }^{+} / \mathrm{PI}^{+}$) were determined using a FACSCanto II and FACS Diva software (BD Biosciences, San Diego, CA, USA).

\section{Western blot analysis}

For western blot analysis, neutrophils were lysed with $10 \%$ ice-cold trichloroacetic acid to prevent protein degradation by neutrophil proteases, as described previously. ${ }^{59}$

Proteins were separated by reducing SDS-PAGE, transferred to a nitrocellulose membrane and blocked for $30 \mathrm{~min}$ at room temperature with TBST containing 5\% BSA. The membranes were then probed with primary Abs (human/mouse/rat MIF Ab, 1:800; human IL-16 C-terminal peptide Ab, $1: 1333$; human S100A8 Ab, $1: 800$ or human/mouse caspase-3 $\mathrm{Ab}, 1: 400$; purchased from R\&D Systems) at $4{ }^{\circ} \mathrm{C}$ overnight. After washing, the membranes were probed with the corresponding secondary Abs (sheep IgG HRP-conjugated Ab, 1:1000; goat IgG HRP-conjugated Ab, $1: 1000$, R\&D Systems) for $1 \mathrm{~h}$ at room temperature. After washing, the membranes were soaked with Immobilon Western chemiluminescent HRP substrate (Millipore, Billerica, MA, USA). Signals of binding Abs were detected with the Fusion FX7 chemiluminescence reader and quantified using the Bio1D software (Vilber Lourmat, Eberhardzell, Germany). Equal loading of the gel was determined by reprobing the membranes with GAPDH (14C10) rabbit HRP-conjugated Ab, 1:1000 (Cell Signaling Technologies).

\section{ELISA for IL-16 and MIF detection}

Concentrations of human IL-16 and MIF in the supernatants were quantified using commercial ELISA kits (DuoSets, R\&D Systems) according to the manufacturer's instructions. According to the manufacturer's information, the antibodies used in the human IL-16 DuoSet are directed against rhIL-16C (\#AAC12732). Nevertheless, cross-reactivity with pre-IL-16 cannot be excluded, as the manufacturer did not test the detection of pre-IL-16. Assuming no resynthesis of pre-IL-16 during apoptosis, our own testing, using lysates (as prepared in the cytokine array) of $5 \times 10^{6}$ freshly isolated and MACS-enriched apoptotic neutrophils, indicates a 3.7 times stronger recognition of IL-16C than of pre-IL-16 (mean $710.9 \mathrm{pg} / \mathrm{ml}$ versus mean $192.6 \mathrm{pg} / \mathrm{ml}, n=3, P=0.040$ as calculated by $t$-test for paired samples).

\section{UV-light-induced apoptosis}

To facilitate neutrophil apoptosis, $350 \mu \mathrm{l}$ of $7 \times 10^{6}$ freshly isolated neutrophils were irradiated with $256 \mathrm{~nm}$ wavelength UV light (200$1600 \mathrm{~mJ} / \mathrm{cm}^{2}$ ) using a Stratalinker (Stratagene, Heidelberg, Germany). Subsequently, the irradiated cells were incubated at a concentration of $5 \times 10^{6}$ cells $/ \mathrm{ml}$ in complete medium for $6 \mathrm{~h}$ at $37^{\circ} \mathrm{C}$. 
Preparation of cell-free A. phagocytophilum and co-incubation with neutrophils

The infection rate of $A$. phagocytophilum-exposed $\mathrm{HL}-60$ cells was determined by counting the cells containing morulae in Diff-Quikstained cytospin preparations. When $\geq 40 \%$ of the cells were found to be infected, they were used for the preparation of cell-free A. phagocytophilum, as described previously. ${ }^{60}$ Briefly, infected HL-60 cells were centrifuged at a speed of $250 \times g$ for $10 \mathrm{~min}$, resuspended in $2 \mathrm{ml}$ of PBS and passed through a 25-gauge needle 10-14 times. Cellular debris was removed by pelleting at $750 \times g$ for $10 \mathrm{~min}$. The supernatant was collected and centrifuged at $2500 \times g$ for $15 \mathrm{~min}$. A . phagocytophilum obtained in this way was immediately resuspended in neutrophil suspension and incubated for $6 \mathrm{~h}$ at $37^{\circ} \mathrm{C}$. For the infection of neutrophils, A. phagocytophilum derived from one infected $\mathrm{HL}-60$ cell was used to infect one PMN. ${ }^{31}$

\section{ABBREVIATIONS}

Ab, antibody; A. phago., Anaplasma phagocytophilum; AU, arbitrary units; DAMPs, danger-associated molecular pattern molecules; IL-16, interleukin-16; LDH, lactate dehydrogenase; MIF, macrophage migration inhibitory factor; $\mathrm{Pl}$, propidium iodide.

\section{ACKNOWLEDGEMENTS}

This work was supported by the Research Training Group 'Modulation of Autoimmunity' (DFG GKR1727/1). We thank Dr. Kai Kalies (Institute of Biology, University of Lübeck) for the technical assistance with ultracentrifugation.

\section{COMPETING INTERESTS}

The authors declare no conflict of interest.

\section{REFERENCES}

1 Saverymuttu SH, Peters AM, Keshavarzian A, Reavy HJ, Lavender JP. The kinetics of 111 indium distribution following injection of 111 indium labelled autologous granulocytes in man. Br J Haematol 1985; 61: 675-685.

2 McCracken JM, Allen L-AH. Regulation of human neutrophil apoptosis and lifespan in health and disease. J Cell Death 2014; 7: 15-23.

3 Silva MT. Secondary necrosis: the natural outcome of the complete apoptotic program. FEBS Lett 2010; 584: 4491-4499.

4 Muñoz LE, Lauber K, Schiller M, Manfredi AA, Herrmann M. The role of defective clearance of apoptotic cells in systemic autoimmunity. Nat Rev Rheumatol 2010; 6 : 280-289.

5 Denecker G, Vercammen D, Steemans M, Vanden Berghe T, Brouckaert G, Van Loo $G$ et al. Death receptor-induced apoptotic and necrotic cell death: differential role of caspases and mitochondria. Cell Death Differ 2001; 8: 829-840.

$6 \mathrm{Wu}$ X, Molinaro C, Johnson N, Casiano CA. Secondary necrosis is a source of proteolytically modified forms of specific intracellular autoantigens: implications for systemic autoimmunity. Arthritis Rheum 2001; 44: 2642-2652.

7 Kolaczkowska E, Kubes P. Neutrophil recruitment and function in health and inflammation. Nat Rev Immunol 2013; 13: 159-175.

8 Amulic B, Cazalet C, Hayes GL, Metzler KD, Zychlinsky A. Neutrophil function: from mechanisms to disease. Annu Rev Immunol 2012; 30: 459-489.

9 Sheshachalam A, Srivastava N, Mitchell T, Lacy P, Eitzen G. Granule protein processing and regulated secretion in neutrophils. Front Immunol 2014; 5: 448.

10 Borregaard N. Neutrophils, from marrow to microbes. Immunity 2010; 33: 657-670.

11 Pellmé $\mathrm{S}$, Mörgelin $\mathrm{M}$, Tapper $\mathrm{H}$, Mellqvist $\mathrm{U}-\mathrm{H}$, Dahlgren $\mathrm{C}$, Karlsson $\mathrm{A}$. Localization of human neutrophil interleukin-8 (CXCL-8) to organelle(s) distinct from the classical granules and secretory vesicles. J Leukoc Biol 2006; 79 : 564-573.

12 Matzer SP, Baumann T, Lukacs NW, Röllinghoff M, Beuscher HU. Constitutive expression of macrophage-inflammatory protein 2 (MIP-2) mRNA in bone marrow gives rise to peripheral neutrophils with preformed MIP-2 protein. $J$ Immunol 2001; 167: 4635-4643.

13 Faurschou M, Borregaard N. Neutrophil granules and secretory vesicles in inflammation. Microbes Infect 2003; 5: 1317-1327.

14 Daryadel A, Grifone RF, Simon H-U, Yousefi S. Apoptotic neutrophils release macrophage migration inhibitory factor upon stimulation with tumor necrosis factor-alpha. J Biol Chem 2006; 281: 27653-27661.

15 Terebuh PD, Otterness IG, Strieter RM, Lincoln PM, Danforth JM, Kunkel SL et al. Biologic and immunohistochemical analysis of interleukin- 6 expression in vivo.
Constitutive and induced expression in murine polymorphonuclear and mononuclear phagocytes. Am J Pathol 1992; 140: 649-657.

16 Bliss SK, Butcher BA, Denkers EY. Rapid recruitment of neutrophils containing prestored IL-12 during microbial infection. J Immunol 2000; 165: 4515-4521.

17 Brandt E, Woerly G, Younes AB, Loiseau S, Capron M. IL-4 production by human polymorphonuclear neutrophils. J Leukoc Biol 2000; 68: 125-130.

18 Beil WJ, Weller PF, Peppercorn MA, Galli SJ, Dvorak AM. Ultrastructural immunogold localization of subcellular sites of TNF-alpha in colonic Crohn's disease. J Leukoc Biol 1995; 58: 284-298.

19 Calafat J, Janssen $H$, Ståhle-Bäckdahl M, Zuurbier AE, Knol EF, Egesten A. Human monocytes and neutrophils store transforming growth factor-alpha in a subpopulation of cytoplasmic granules. Blood 1997; 90: 1255-1266.

20 Schröder AK, von der Ohe M, Kolling U, Altstaedt J, Uciechowski P, Fleischer D et al. Polymorphonuclear leucocytes selectively produce anti-inflammatory interleukin-1 receptor antagonist and chemokines, but fail to produce proinflammatory mediators. Immunology 2006; 119: 317-327.

21 Borregaard N, Sørensen OE, Theilgaard-Mönch K. Neutrophil granules: a library of innate immunity proteins. Trends Immunol 2007; 28: 340-345.

22 Flieger O, Engling A, Bucala R, Lue H, Nickel W, Bernhagen J. Regulated secretion of macrophage migration inhibitory factor is mediated by a non-classical pathway involving an $A B C$ transporter. FEBS Lett 2003; 551: 78-86.

23 Zhang Y, Center DM, Wu DM, Cruikshank WW, Yuan J, Andrews DW et al. Processing and activation of pro-interleukin-16 by caspase-3. J Biol Chem 1998; 273: 1144-1149.

24 Baier M, Bannert N, Werner A, Lang K, Kurth R. Molecular cloning, sequence, expression, and processing of the interleukin 16 precursor. Proc Natl Acad Sci USA 1997; 94: 5273-5277.

25 Richmond J, Tuzova M, Cruikshank W, Center D. Regulation of Cellular Processes by Interleukin-16 in Homeostasis and Cancer. J Cell Physiol 2014; 229: 139-147.

26 Wu DM, Zhang Y, Parada NA, Kornfeld H, Nicoll J, Center DM et al. Processing and release of IL-16 from CD4+ but not CD8+ T cells is activation dependent. J Immunol 1999; 162: 1287-1293.

27 Liu Y, Cruikshank WW, O'Loughlin T, O'Reilly P, Center DM, Kornfeld H. Identification of a CD4 domain required for interleukin-16 binding and lymphocyte activation. J Biol Chem 1999; 274: 23387-23395.

28 Lynch EA, Heijens CAW, Horst NF, Center DM, Cruikshank WW. Cutting edge: IL-16/CD4 preferentially induces Th1 cell migration: requirement of CCR5. J Immunol 2003; 171: 4965-4968.

29 Scheel-Toellner D, Wang K, Craddock R, Webb PR, McGettrick HM, Assi LK et al. Reactive oxygen species limit neutrophil life span by activating death receptor signaling. Blood 2004; 104: 2557-2564.

30 Han Z, Hendrickson EA, Bremner TA, Wyche JH. A sequential two-step mechanism for the production of the mature p17:p12 form of caspase-3 in vitro. $J$ Biol Chem 1997: 272: 13432-13436.

31 Sarkar A, Hellberg L, Bhattacharyya A, Behnen M, Wang K, Lord JM et al. Infection with Anaplasma phagocytophilum activates the phosphatidylinositol 3-Kinase/ Akt and NF-KB survival pathways in neutrophil granulocytes. Infect Immun 2012; 80: $1615-1623$.

32 Tecchio C, Micheletti A, Cassatella MA. Neutrophil-derived cytokines: facts beyond expression. Front Immunol 2014; 5: 1-7.

33 Gaudry M, Brégerie O, Andrieu V, El Benna J, Pocidalo MA, Hakim J. Intracellular pool of vascular endothelial growth factor in human neutrophils. Blood 1997; 90 4153-4161.

34 Kavanagh E, Rodhe J, Burguillos MA, Venero JL, Joseph B. Regulation of caspase-3 processing by CIAP2 controls the switch between pro-inflammatory activation and cell death in microglia. Cell Death Dis 2014; 5: e1565.

35 Hessianl PA, Edgeworth J, Hogg N. MRP-8 and MRP-14, two abundant $\mathrm{Ca}(2+)$-binding proteins of neutrophils and monocytes. J Leukoc Biol 1993; 53 : 197-204

36 Lopez-Castejon G, Brough D. Understanding the mechanism of IL-1 $\beta$ secretion. Cytokine Growth Factor Rev 2011; 22: 189-195.

37 Steringer JP, Müller H-M, Nickel W. Die molekulare Entschlüsselung unkonventioneller Sekretionsmechanismen. BIOspektrum 2014; 20: 400-403.

38 Katano M, Okamoto K, Suematsu N, Kurokawa MS, Nakamura H, Masuko K et al. Increased expression of $\mathrm{S} 100$ calcium binding protein A8 in GM-CSF-stimulated neutrophils leads to the increased expressions of IL-8 and IL-16. Clin Exp Rheumatol 2011; 29: 768-775.

39 Riedemann NC, Guo R-F, Gao H, Sun L, Hoesel M, Hollmann TJ et al. Regulatory role of $\mathrm{C} 5$ a on macrophage migration inhibitory factor release from neutrophils. J Immunol 2004; 173: 1355-1359.

40 Riedemann NC, Guo R-F, Gao H, Sun L, Hoesel M, Hollmann TJ et al. Regulatory role of $\mathrm{C} 5 \mathrm{a}$ on macrophage migration inhibitory factor release from neutrophils. J Immunol 2004; 173: 1355-1359. 
41 Calandra T, Bernhagen J, Metz CN, Spiegel LA, Bacher M, Donnelly T et al. MIF as a glucocorticoid-induced modulator of cytokine production. Nature 1995; 377: 68-71.

42 Ruiz LM, Bedoya G, Salazar J, García de OD, Patiño PJ. Dexamethasone inhibits apoptosis of human neutrophils induced by reactive oxygen species. Inflammation 2002; 26: 215-222.

43 Klebanoff SJ, Coombs RW. Viricidal effect of polymorphonuclear leukocytes on human immunodeficiency virus-1. Role of the myeloperoxidase system. J Clin Invest 1992; 89: 2014-2017.

44 Pitrak DL, Tsai HC, Mullane KM, Sutton SH, Stevens P. Accelerated neutrophil apoptosis in the acquired immunodeficiency syndrome. J Clin Invest 1996; 98: 2714-2719.

45 Torre D, Gennero L, Baccino FM, Speranza F, Biondi G, Pugliese A. Impaired macrophage phagocytosis of apoptotic neutrophils in patients with human immunodeficiency virus type 1 infection. Clin Diagn Lab Immunol 2002; 9: 983-986.

46 Idziorek T, Khalife J, Billaut-Mulot O, Hermann E, Aumercier M, Mouton $\mathrm{Y}$ et al. Recombinant human IL-16 inhibits HIV-1 replication and protects against activation-induced cell death (AICD). Clin Exp Immunol 1998; 112: 84-91.

47 Amiel C, Darcissac E, Truong MJ, Dewulf J, Loyens M, Mouton Y et al. Interleukin16 (IL-16) inhibits human immunodeficiency virus replication in cells from infected subjects, and serum IL-16 levels drop with disease progression. J Infect Dis 1999; 179: 83-91.

48 Shao W, Cohen PL. Disturbances of apoptotic cell clearance in systemic lupus erythematosus. Arthritis Res Ther 2011; 13: 1-7.

49 Ren Y, Tang J, Mok MY, Chan AWK, Wu A, Lau CS. Increased apoptotic neutrophils and macrophages and impaired macrophage phagocytic clearance of apoptotic neutrophils in systemic lupus erythematosus. Arthritis Rheum 2003; 48: 2888-2897.

50 Lard LR, Roep BO, Verburgh CA, Zwinderman AH, Huizinga TWJ. Elevated IL-16 levels in patients with systemic lupus erythematosus are associated with disease severity but not with genetic susceptibility to lupus. Lupus 2002; 11: 181-185.

51 Lee S, Kaneko H, Sekigawa I, Tokano Y, Takasaki Y, Hashimoto H. Circulating interleukin-16 in systemic lupus erythematosus. $\mathrm{Br} J$ Rheumatol 1998; 37: 1334-1337.
52 Kasama T, Ohtsuka K, Sato M, Takahashi R, Wakabayashi K, Kobayashi K. Macrophage migration inhibitory factor: a multifunctional cytokine in rheumatic diseases. Arthritis 2010; 2010: 106202.

53 Ayoub S, Hickey MJ, Morand EF. Mechanisms of disease: macrophage migration inhibitory factor in SLE, RA and atherosclerosis. Nat Clin Pract Rheumatol 2008; 4: 98-105.

54 Santos LL, Fan H, Hall P, Ngo D, Mackay CR, Fingerle-Rowson G et al. Macrophage migration inhibitory factor regulates neutrophil chemotactic responses in inflammatory arthritis in mice. Arthritis Rheum 2011; 63: 960-970.

55 Gregory JL, Morand EF, McKeown SJ, Ralph JA, Hall P, Yang YH et al. Macrophage migration inhibitory factor induces macrophage recruitment via CC chemokine ligand 2. J Immunol 2006; 177: 8072-8079.

56 Aga E, Katschinski DM, van Zandbergen G, Laufs H, Hansen B, Müller K et al. Inhibition of the spontaneous apoptosis of neutrophil granulocytes by the intracellular parasite Leishmania major. J Immunol 2002; 169: 898-905.

57 Kjeldsen L, Sengelov H, Borregaard N. Subcellular fractionation of human neutrophils on Percoll density gradients. J Immunol Methods 1999; 232: 131-143.

58 Udby L, Borregaard N. Subcellular fractionation of human neutrophils and analysis of subcellular markers. Methods $\mathrm{Mol}$ Biol 2007; 412: 35-56.

59 Hellberg L, Samavedam UKSRL, Holdorf K, Hänsel M, Recke A, Beckmann T et al. Methylprednisolone blocks autoantibody-induced tissue damage in experimental models of bullous pemphigoid and epidermolysis bullosa acquisita through inhibition of neutrophil activation. J Invest Dermatol 2013; 133: 2390-2399.

60 Choi K-S, Park JT, Dumler JS. Anaplasma phagocytophilum delay of neutrophil apoptosis through the p38 mitogen-activated protein kinase signal pathway. Infect Immun 2005; 73: 8209-8218.

(c) (i) This work is licensed under a Creative Commons Attribution 4.0 article are included in the article's Creative Commons license, unless indicated otherwise in the credit line; if the material is not included under the Creative Commons license, users will need to obtain permission from the license holder to reproduce the material. To view a copy of this license, visit http://creativecommons.org/licenses/ by/4.0/

Supplementary Information accompanies the paper on the Cell Death Discovery website (http://www.nature.com/cddiscovery) 